\title{
Correlation between Ethylene Adsorption Energies and Core-Level Shifts for Pd Nanoclusters
}

Alexey A. Tal, Alvaro Posada-Borbon, Henrik J . Gronbeck and Igor Abrikosov

The self-archived postprint version of this journal article is available at Linköping University Institutional Repository (DiVA):

http:/ / urn.kb.se/ resolve?urn=urn:nbn:se:liu:diva-154685

N.B.: When citing this work, cite the original publication.

Tal, A. A., Posada-Borbon, A., Gronbeck, H. J ., Abrikosov, I., (2019), Correlation between Ethylene Adsorption Energies and Core-Level Shifts for Pd Nanoclusters, The J ournal of Physical Chemistry C, 123(4), 2544-2548. https:// doi.org/ 10.1021/ acs.jpcc.8b10465

Original publication available at:

https:// doi.org/ 10.1021/acs.jpcc.8b10465

Copyright: AMER CHEMICAL SOC

https:// www.acs.org/ 


\title{
Correlation Between Ethylene Adsorption Energies and Core-Level Shifts for Pd Nanoclusters
}

\author{
Alexey A. Tal, ${ }^{* \dagger \dagger}$ Alvaro Posada-Borbón, ${ }^{\ddagger}$ Henrik J. Grönbeck,${ }^{\ddagger}$ and Igor A. \\ Abrikosov ${ }^{\top, \S}$ \\ $\dagger$ †haire de Simulation à l'Echelle Atomique (CSEA), Ecole Polytechnique Fédérale de \\ Lausanne (EPFL), CH-1015 Lausanne, Switzerland \\ $\ddagger$ Department of Physics and Competence Centre for Catalysis, Chalmers University of \\ Technology, SE-41296 Göteborg, Sweden \\ \Department of Physics, Chemistry and Biology (IFM), Linköping University, SE-581 83, \\ Linköping, Sweden \\ $\S$ Materials Modeling and Development Laboratory, National University of Science and \\ Technology 'MISIS', 119049, Moscow, Russia \\ E-mail: alexey.tal@epfl.ch, alyxthal@gmail.com
}

\begin{abstract}
Density Functional Theory calculations have been used to investigate adsorption of ethylene on $\mathrm{Pd}$ nanocluster together with shifts in core-level binding energies of Pd-atoms bonded to the adsorbate. The adsorption energy is found to correlate with the core-level shifts (CLS) which is consistent with the notion that core-level binding energies is a measure of differences in cohesion. The correlation between adsorption energies and core-level shifts is found to be stronger than the correlation between
\end{abstract}


adsorption energies and generalized coordination numbers, indicating that descriptors preferably should account for electronic effects explicitly. The advantages of CLS as a descriptor for a screening of adsorption properties is discussed.

\section{Introduction}

The unique properties of nanoclusters and applications within heterogeneous catalysis ${ }^{1}$, photovoltaics $^{2}$, quantum $\operatorname{dots}^{3}$ and plasmonics ${ }^{4}$ explains and motivates the large amount of work directed to fundamental understanding of finite-size systems. It is well established that properties of nanoclusters (NCs) are determined both by geometric effects related to undercoordinated surface atoms and to consequences of electronic confinement. The importance of electron confinement is generally most pronounced in the, so-called, non-scalable regime with particle diameters below $\sim 2 \mathrm{~nm} . \underline{[5}$

The large surface-to-volume ratio makes metal NC important in heterogeneous catalysis. Such catalysts are generally realized as precious metal NC dispersed on porous oxide supports. Precise control and understanding of NC properties is one route to improve the reactivity of catalytic systems. As explicit treatments of NC reactivity and, eventually, catalytic activity from first principles are computationally demanding, different types of descriptors have been developed over the past decades. The descriptors are commonly based on either the local electronic structure or the geometric structure through atomic coordination. One benefit of descriptors, is that predictions are linked to chemical understanding, another is that they can be used for screening of catalytic activity. ${ }^{617}$

A descriptor should preferably be easy to calculate allowing for efficient exploration of multiple systems. One seminal example is the $d$-band model,,$[9]$ which relates adsorption energies $E_{a d s}$ on transition metals by the position of the $d$-band relative to the Fermi level. A number of additional descriptors based on electronic structure have been discussed over the years such as vacancy formation energy, ionization potential and electron affinity. 10 The electronic structure reflects the atomic structure which explains why descriptors based solely 
on the local atomic structure also show a good performance. One such example is the recent work by Calle-Vallejo et al. suggesting that the generalized coordination number (GCN) calculated from the local coordination provides a good measure of adsorption energies.11 Unlike regular coordination numbers, GCN takes into account the second coordination shell and, thus, includes more information on the electronic properties. Moreover, an orbitalwise coordination number $\left(C N^{\alpha}\right)$ calculated as two-center s-electrons hopping integrals to neighboring atoms was recently suggested by Ma and co-workers. 12

The position of the $d$-band relative the Fermi energy is to a large extent determined by the electrostatic potential at the considered site and the same applies to core-states. $\frac{13}{1}$ Thus, the adsorption energy should correlate also with the shift of core-state binding energies, as measured in X-ray photoemission spectroscopy (XPS). This has been demonstrated for

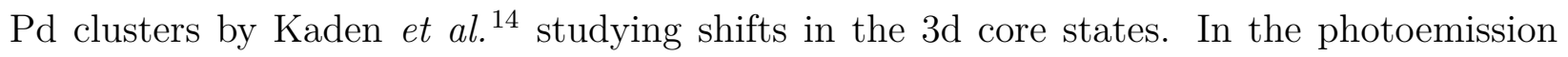
process, an electron from a core-level is emitted and a hole is created which affects the remaining electrons. The shift of the core-electron binding energies without the screening effects induced by the hole is generally referred to as the initial state shift. However, since the measured binding energies include the effects of the hole, it should be accounted for in computational treatments. Shifts calculated including the screening of the core-hole are called final state shifts.

Density functional theory (DFT) calculations have shown to accurately reproduce measured core level shifts (CLS) for adsorption of simple adsorbates on metal surfaces. $\frac{1516}{16}$ Recent work also include metal NC ${ }^{17 \mid 18}$ and in Ref. ${ }^{18}$ it was shown how confinement effects, local coordination and interatomic distances affect the $4 \mathrm{f}$ CLS for bare Au NC. As of yet, however, the dependence of CLS on adsorption energies has not been comprehensively investigated for metal NC. In this work, we use density functional theory (DFT) calculations to study ethylene $\left(\mathrm{C}_{2} \mathrm{H}_{4}\right)$ adsorption on Pd NC. Ethylene hydrogenation is an important model reaction with bearing on, for example, upgrading of biofuels. $\frac{19}{}$ Previous studies have shown that ethylene hydrogenation starts with ethylene adsorbed atop a metal atoms, the so-called 
$\pi$-mode. ${ }^{20-\sqrt[22]{22}}$ Here, we restrict the treatment to this adsorption mode on either icosahedral (Ih) and truncated octahedral (TOh) Pd NC.

\section{Computational details}

The DFT calculations were performed with the Vienna ab-initio simulation package, VASP ${ }^{23}$ using the spin-restricted PBE exchange-correlation functional. ${ }^{24}$ The Kohn-Sham orbitals were expanded with plane waves using an energy cut-off of $420 \mathrm{eV}$ and the interaction between the valence electrons and the cores was described with the plane augmented wave (PAW) method. ${ }^{25}$ The structures were relaxed until all forces were $<0.05 \mathrm{eV} / \AA$. All clusters were separated by at least $10 \AA$ of vacuum from their periodic images. The Fermi discontinuity was smeared applying the Methfessel and Paxton scheme ${ }^{26}$ with a smearing width of 0.05 $\mathrm{eV}$. As references we considered extended $\mathrm{Pd}(100)$ and $\mathrm{Pd}(111)$ with $\mathrm{p}(3 \mathrm{x} 3)$ and $\mathrm{p}(4 \mathrm{x} 4)$ surface cells, respectively. Integration over the Brillouin zone was approximated by finite sampling using a k-point grid of $(6 \times 6 \times 1)$ for both surfaces.

The initial state CLS were evaluated by solving the Kohn-Sham equations for core electrons perturbatively, after self-consistency with frozen core electrons had been attained 27. The CLS was in this case obtained as differences in the Kohn-Sham eigenvalues of the 3d state. For the CLS calculations with final state effects, the so-called Z+1 approach 28 was used in which it is assumed that an ionized atom can be effectively treated as a neutral atom with an extra proton. The charging of the system were neglected and the CLS were obtained as total energy differences. This approach has been shown to give accurate results for atoms, molecules, surfaces and extended systems. $\frac{15128}{31}$

Within the Z+1 approach, the CLS can be computed as a difference of ionization energies, i.e. the difference between ground state total energy and the total energy of the core-ionized state. Thus, it is convenient to introduce a generalized thermodynamic chemical potential 
$(\mathrm{GTCP})^{28}$

$$
\mu=\frac{E^{i o n}-E^{g s}}{1 / N}
$$

where $\mu$ is the GTCP and $\mathrm{N}$ is the number of ionized atoms in the supercell $(N=1$ in our calculations). The energies $E^{g s}$ and $E^{i o n}$ are the total energies of the system in the ground state and ionized states, respectively. The CLS are the difference between GTCP of the reference state and the system of interest:

$$
E_{C L S}=\Delta \mu_{i}=\mu_{i}-\mu_{i}^{R e f}
$$

Here, we use bulk fcc $\mathrm{Pd}$ as a reference system and all the energies are calculated relative to the corresponding Fermi level. The calculations of the CLS in bulk were converged using a supercell size of $3 \times 3 \times 3$ unit cells. At this size, the interaction of an ionized atom with its periodic image were negligibly small.

We have studied Pd nanoclusters with octahedral (TOh) and icosahedral (Ih) morpholo-

gies. These two shapes are representative for small metal NC. ${ }^{32}$ In particular, we have considered TOh clusters with 13, 38, 79 and 116 atoms and Ih clusters with 13 and 55 atoms.

To calculate the adsorption energy of ethylene on the Pd NC, three energies are calculated; one for the optimized bare particle, one for the gas phase ethylene and one for the case when the molecule is bound to the particle and the geometry is optimized, as shown in Fig. 1. The energy of the combined system minus the sum of the energies of the bare particle and ethylene molecule is the adsorption energy.

\section{Results and discussions}

Here we explore the relation between ethylene adsorption energies on Pd NC and their CLS calculated in both the initial and the final state approximations. 
Table 1: Ethylene adsorption energies $\left(E_{a d s}\right)$ and generalized coordination number $(\mathrm{GCN})$ for the considered site. Ethylene adsorption is considered in the $\pi$-mode. CLS $_{\text {init }}$ and CLS $_{\text {final }}$ denote core-level shifts calculated in the initial and final state approximations, respectively. The center of the d-band with respect to the Fermi-energy is denoted $d$-band. All energies are reported in $\mathrm{eV}$.

\begin{tabular}{crrrrrr} 
Cluster & Site & $E_{a d s}$ & GCN & CLS $_{\text {init }}$ & CLS $_{\text {final }}$ & $d$-band \\
\hline \hline Pd13TOh & & -1.58 & 2.67 & -1.31 & -0.88 & -0.65 \\
Pd38TOh & A & -0.67 & 6.00 & -0.41 & -0.24 & -0.18 \\
Pd38TOh & B & -1.18 & 4.00 & -0.80 & -0.47 & -0.33 \\
Pd79TOh & A & -1.13 & 4.08 & -0.75 & -0.37 & -0.29 \\
Pd79TOh & B & -1.12 & 5.00 & -0.67 & -0.48 & -0.32 \\
Pd79TOh & C & -0.78 & 6.67 & -0.35 & -0.25 & -0.15 \\
Pd116TOh & A & -1.16 & 4.00 & -0.80 & -0.45 & -0.39 \\
Pd116TOh & C & -1.15 & 5.17 & -0.55 & -0.32 & -0.27 \\
Pd116TOh & B & -0.89 & 6.33 & -0.40 & -0.20 & -0.20 \\
Pd116TOh & D & -0.91 & 6.67 & -0.38 & -0.30 & -0.23 \\
Pd13Ih & & -1.52 & 3.50 & -1.07 & -0.81 & -0.53 \\
Pd55Ih & B & -1.36 & 4.33 & -0.85 & -0.50 & -0.31 \\
Pd55Ih & A & -1.15 & 5.67 & -0.78 & -0.47 & -0.39 \\
Pd(100) & & -0.89 & 6.67 & -0.56 & -0.29 & -0.21 \\
Pd(111) & & -0.78 & 7.50 & -0.39 & -0.22 & -0.23 \\
\hline \hline
\end{tabular}

The results are presented in Table. 1. Sites considered for adsorption and CLS are marked with red letters in Fig. 1. Owing to the high symmetry of the nanoclusters, only a limited number of the surface atoms are inequivalent. For the 13-atom cluster, all surface atoms are equivalent. The adsorption energy is highest on the small $\mathrm{N}$, which is in full agreement with a previous report on ethylene adsorption on Pd clusters. $\frac{33}{33}$ The highest adsorption energy for a particular NC is generally obtained on the least coordinated atoms, e.g. the vertex atom. The largest amount of the adsorption energy is related to the direct adsorbate-metal interaction. The structural relaxation energies are in all cases found to be less than $0.15 \mathrm{eV}$, where the largest relaxation energies are calculated for the smallest clusters.

The CLS show a strong size dependence, with large shifts calculated for the smallest clusters. $\mathrm{Pd}_{13}$ has a high CLS of $-0.88 \mathrm{eV}$ and $-0.81 \mathrm{eV}$ for the octahedral and icosahedral geometries, respectively. The values for the clusters are generally higher than for the extended $\operatorname{Pd}(100)$ and $\operatorname{Pd}(111)$ surfaces. The experimental values for $\operatorname{Pd}(100)$ and $\operatorname{Pd}(111)$ are -0.44 


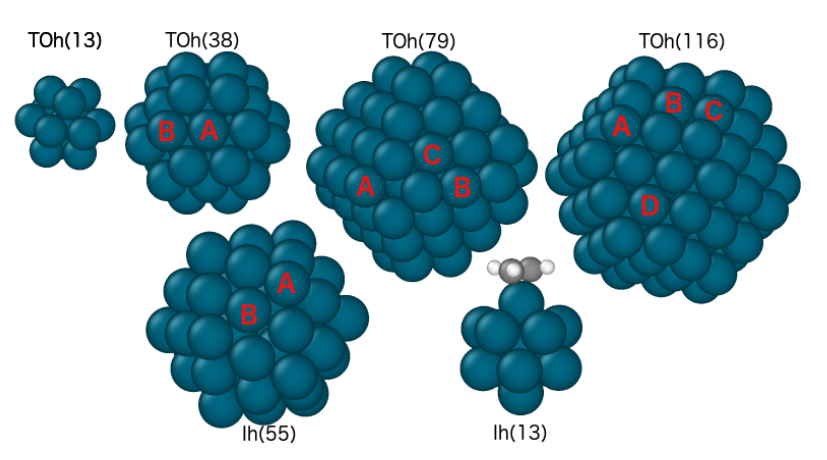

Figure 1: Structures of Pd particles with tetrahedral (TOh) and icosahedral (Ih) morphologies. The different sites for which CLS and $E_{a d s}$ were calculated, are indicated by capital letters A, B and C. $\pi$-mode adsorption of ethylene is exemplified for a 13-atom icosahedral cluster.

and $-0.28 \mathrm{eV}$, respectively. ${ }^{34}$ The final states effects in the $\mathrm{NC}$ were found to be most (least) pronounced for vertex (facet) atoms.

Frequently, factors based on local geometry are used as descriptors for adsorption energies. For example, generalized coordination number accounts for the number of neighbors with their coordinations. However, as exemplified in Ref. 18 , the size-evolution of electronic properties of small nanoclusters is non-monotonous and highly sensitive to confinement effects and local strains. ${ }^{35136}$ Thus, the shifts of the valence band, core states and adsorption energy can generally not be fully described by descriptors that explicitly do not account for such effects. In Fig. 2 the correlation between adsorption energy and GCN is shown. The correlation coefficient $R^{2}$ is 0.79 and the distribution of the data points is rather broad. This indicates that there is a room for improvement, even though GCN is fair descriptor.

When correlating the adsorption energies instead to the CLS, some effects that are missing in geometric approach are included, yielding a better correlation. As shown in Fig. 3, a good correlation is observed for the full range of considered sizes and the correlation coefficient $R_{C S}^{2}$ is 0.89 . Thus, CLS in initial state approximation show improved correlation as compared to GCN.

Chemical analysis is based on the assumption that the measured CLS depends on the electrostatic potential at the site under consideration. $\frac{37}{37}$ However, measurement also includes 


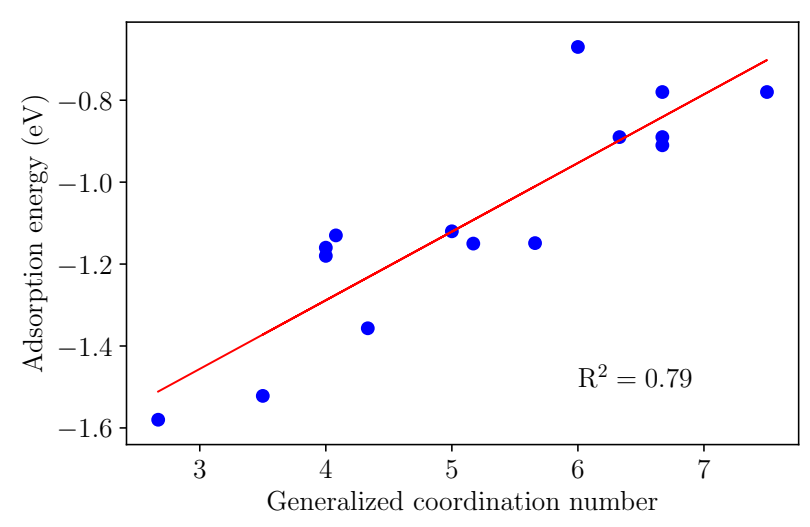

Figure 2: Adsorption energies of ethylene on $\mathrm{Pd} \mathrm{NC}$ as a function of generalized coordination number.

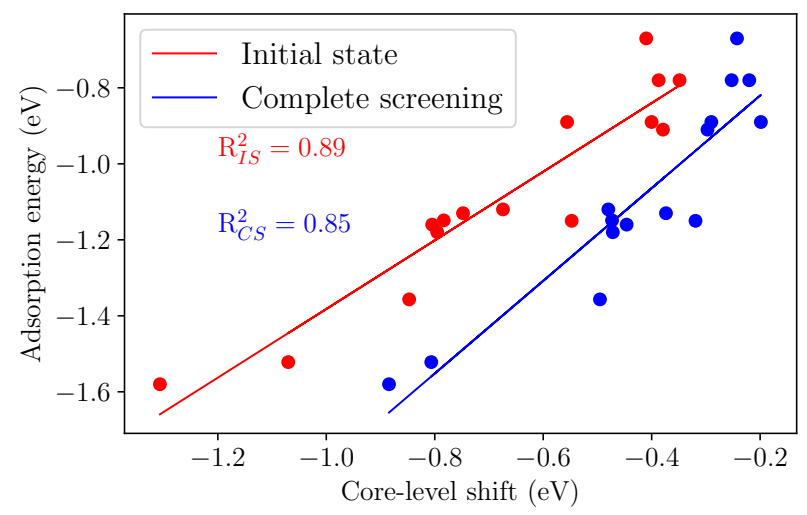

Figure 3: Adsorption energy of ethylene on Pd NC as a function of CLS calculated within initial and final state approximations.

final state effects of the core hole is screened. The potential model suggested by Gelius ${ }^{38}$ implies that CLS are approximated by the change of the electrostatic potential for different local environments $\Delta V$ with a correction for relaxation effects due to the presence of a core-hole $\Delta E_{R}$ :

$$
E_{C L S}^{p o t}=\Delta V-\Delta E_{R}
$$

The effect of final state effects on the correlation between $E_{a d s}$ and CLS is shown in Fig 3 The values that are furthest from the linear fit, correspond generally to vertex atoms. The correlation is strong $\left(R_{I S}^{2}=0.85\right)$ and close to the calculations including only the initial state contribution. 
According to the $d$-band model,${ }^{8}$ the difference of adsorption energies on transition metals in different environments can be determined by the location of the $d$-band center with respect to the Fermi-energy. In previous work, it was shown that CLS in nanoclusters correlate with differences in $d$-band centers. $\frac{18}{18}$ This is understandable as the $d$-band center shifts, in similarity with the shifts of the core states eigenvalues, are determined by the shift of the electrostatic potential. In Table 1 the values for $d$-band centers were calculated as the first moment of the projected $d$-band density of states referenced to the Fermi level. In the left panel of Fig. 4 the discrepancies in correlation between initial-state CLS and d-band shifts can be attributed to the difference in the electrostatic potential shift for states with different localization of the wave function, as discussed in Ref. ${ }^{[39}$ In the right panel of Fig. 4 the correlation for complete screening CLS and initial-state CLS is shown and the deviation from a linear correspondence is due to the effects of the core-hole screening. The correlation between the adsorption energies and the shifts of the $d$-band centers is also clear although the correlation is slightly worse than in the case of the core-level shifts, as shown in Fig. 5 .
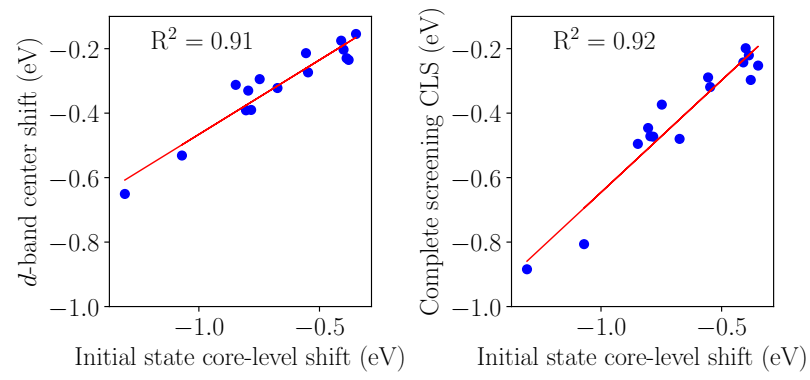

Figure 4: Complete screening CLS and $d$-band center shifts as a function of initial state CLS.

The analysis of CLS in small nanoclusters presented in Ref. ${ }^{18}$ suggests that the final state effects partially compensate for the initial state shift (see Eq. 3), typically decreasing the absolute value of the shift. Nevertheless, the effect of the core-hole screening is rather modest and similar for different sizes and structures. The deviation from the scaling relation does not exceed $0.1 \mathrm{eV}$. The points that deviate the most, typically correspond to vertex (the most undercoordinated) atoms. As shown in Fig. 4, the correlation is strong, meaning 
that CLS can be potentially used as a descriptor for reactivity of small nanoclusters.

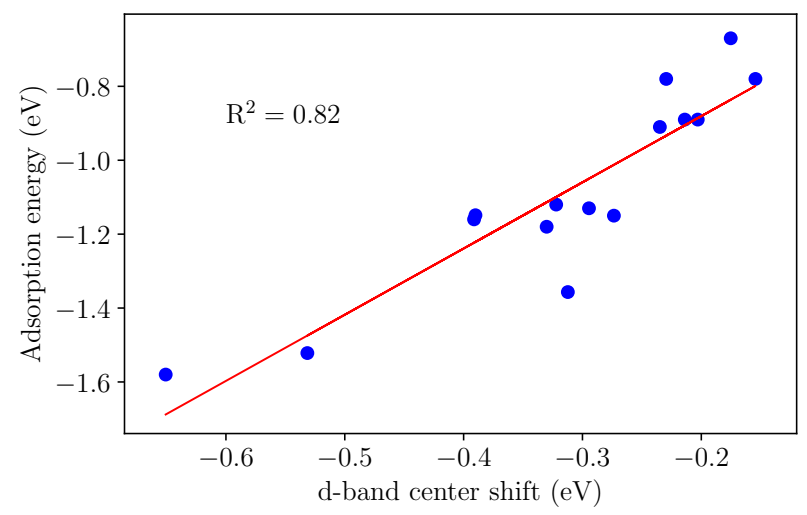

Figure 5: Adsorption energy of an ethylene molecule on the surface of $\mathrm{Pd}$ nanoclsuters as a function of $d$-band center shift.

\section{Conclusions}

We have investigated ethylene adsorption and CLS for the unique atoms in the icosahedral and octahedral Pd nanoclusters. We find that the ethylene adsorption energy correlates with CLS calculated either in the initial or final state approximation. These correlations are just as strong as the correlation between the adsorption energies and the $d$-band centers. Inclusion of final state effects in the CLS is found to slightly reduce the correlation as compared to CLS obtained in the initial state approximation. However, the final state effects in the CLS are found to be small in all cases. The correlation between the adsorption energy and the generalized coordination number $(\mathrm{GCN})$ is found to be slightly weaker than the CLS and $d$ band center. This is reasonable given that the GCN is a structure-only descriptor not taking, for example, electron confinement effects into account. One particular advantage of CLS as a descriptor is that they can be accurately measured. Regarding computational assessment, evaluation of CLS does not require structural relaxations, which drastically decreases the complexity of calculations, and can be applied also for alloy nanoclusters and supported particles. 


\section{Acknowledgments}

This work was financially supported by the Knut and Alice Wallenberg Foundation through Grant No. 2012.0083 and the Strong Field Physics and New States of Matter 2014-2019 (COTXS). Analysis of the core-level shifts was supported by the Ministry of Education and Science of the Russian Federation in the framework of Increase Competitiveness Program of NUST "MISIS" (No. K2-2019-001) implemented by a governmental decree dated 16 March 2013, No. 211. I.A.A. acknowledges support from the Swedish Government Strategic Research Area in Materials Science on Functional Materials at Linköping University (Faculty Grant SFO-MatLiU No. 2009 00971). The calculations were performed on resources provided by the Swedish National Infrastructure for Computing (SNIC) at NSC (Linköping), C3SE (Göteborg), and the supercomputer cluster provided by the Materials Modeling and Development Laboratory at NUST âĂIJMISISâĂİ. A.P.-B. and H.J.G. acknowledge financial support from the Knut and Alice Wallenberg Foundation through the project Atomistic design of catalysts (No. 2015.0058).

\section{References}

(1) Tyo, E. C.; Vajda, S. Catalysis by Clusters with Precise Numbers of Atoms. Nat. Nanotechnol. 2015, 10, 577-588.

(2) Kovalenko, M. V.; Manna, L.; Cabot, A.; Hens, Z.; Talapin, D. V.; Kagan, C. R.; Klimov, V. I.; Rogach, A. L.; Reiss, P.; Milliron, D. J.; et al., Prospects of Nanoscience with Nanocrystals. ACS Nano 2015, 9, 1012-1057.

(3) Pietryga, J. M.; Park, Y. S.; Lim, J.; Fidler, A. F.; Bae, W. K.; Brovelli, S.; Klimov, V. I. Spectroscopic and Device Aspects of Nanocrystal Quantum Dots. Chem. Rev. 2016, $116,10513-10622$.

(4) Amendola, V.; Pilot, R.; Frasconi, M.; Maragò, O. M.; Iatì, M. A. Surface Plasmon 
Resonance in Gold Nanoparticles: A Review. J. Phys. Condens. Matter 2017, 29, 203002.

(5) Landman, U. Materials by Numbers: Computations as Tools of Discovery. Proc. Natl. Acad. Sci. 2005, 102, 6671.

(6) Jørgensen, M.; Grönbeck, H. Scaling Relations and Kinetic Monte Carlo Simulations to Bridge the Materials-Gap in Heterogeneous Catalysis. ACS Catal. 2017, 7, 5054.

(7) Hoffmann, M.; Bligaard, T. A Lattice Kinetic Monte Carlo Solver for First-Principles Microkinetic Trend Studies. J. Chem. Theory Comput. 2018, 14, 1583-1593.

(8) Nørskov, J. K.; Holloway, S. On the Electronic Factor in Catalysis. Vacuum 1983, 33 , 876.

(9) Hammer, B.; Norskov, J. K. Why Gold is the Noblest of All the Metals. Nature 1995, $376,238-240$.

(10) Capdevila-Cortada, M.; Vilé, G.; Teschner, D.; Pérez-Ramírez, J.; López, N. Reactivity Descriptors for Ceria in Catalysis. Appl. Catal. B Environ. 2016, 197, 299-312.

(11) Viñes, F.; Gomes, J. R. B.; Illas, F. Understanding the Reactivity of Metallic Nanoparticles: Beyond the Extended Surface Model for Catalysis. Chem. Soc. Rev. 2014, 43, 4922-39.

(12) X, M.; Xin, H. Orbitalwise Coordination Number for Predicting Adsorption Properties of Metal Nanocatalysts. Phys. Rev. Lett. 2017, 118, 036101.

(13) Citrin, P.; Wertheim, G. Photoemission from Surface-Atom Core Levels, Surface Densities of States, and Metal-Atom Clusters: A Unified Picture. Phys. Rev. B 1983, 27, $3176-3200$. 
(14) Kaden, W. E.; Wu, T.; Kunkel, W. a.; Anderson, S. L. Electronic Structure Controls Reactivity of Size-Selected Pd Clusters Adsorbed on TiO2 Surfaces. Science 2009, 326, 826-829.

(15) Marten, T.; Olovsson, W.; Simak, S. I.; Abrikosov, I. A. Ab Initio Study of Disorder Broadening of Core Photoemission Spectra in Random Cu-Pd and Ag-Pd Alloys. Phys. Rev. B 2005, 72, 054210.

(16) Nilsson, V.; Van den Bossche, M.; Hellman, A.; Grönbeck, H. Trends in Adsorbate Induced Core Level Shifts. Surf. Sci. 2015, 640, 59-64.

(17) Grönbeck, H. The Bonding in Thiolate Protected Gold Nanoparticles From Au4f Photoemission Core Level Shifts. Nanoscale 2012, 4, 4178.

(18) Tal, A. A.; Olovsson, W.; Abrikosov, I. A. Origin of the Core-Level Binding Energy Shifts in Au Nanoclusters. Phys. Rev. B 2017, 95.

(19) Heard, C. J.; Hu, C.; Skoglundh, M.; Creaser, D.; Grönbeck, H. Kinetic Regimes in Ethylene Hydrogenation over Transition Metal Surfaces. ACS Catalysis 2016, 6, 32773286.

(20) Neurock, M.; Van Santen, R. A. First Principles Analysis of C-H Bond Formation in Ethylene Hydrogenation. J. Phys. Chem. B 2000, 104, 11127-11145.

(21) Heard, C. J.; Siahrostami, S.; Grönbeck, H. Structural and Energetic Trends of Ethylene Hydrogenation over Transition Metal Surfaces. J. Phys. Chem. C 2016, 120, 995-1003.

(22) Ohtani, T.; Kubota, J.; Kondo, J. N.; Hirose, C.; Domen, K. Suppression of Formation of Ethylidyne on Pt(111) by Reversibly Adsorbed di- $\sigma$-bonded Ethylene Studied by In Situ IRAS. Surf. Sci. 1998, 415, L983-L987.

(23) Kresse, G.; Joubert, D. From Ultrasoft Pseudopotentials to the Projector AugmentedWave Method. Phys. Rev. B 1999, 59, 1758-1775. 
(24) Perdew, J. P.; Burke, K.; Ernzerhof, M. Generalized Gradient Approximation Made Simple. Phys. Rev. Lett. 1996, 77, 3865-3868.

(25) Blöchl, P. E. Projector Augmented-Wave Method. Phys. Rev. B 1994, 50, 17953-17979.

(26) Methfessel, M.; Paxton, A. T. High-Precision Sampling for Brillouin-Zone Integration in Metals. Phys. Rev. B 1989, 40, 3616-3621.

(27) Köhler, L.; Kresse, G. Density Functional Study of CO on Rh(111). Phys. Rev. B 2004, $70,165405$.

(28) Johansson, B.; Mårtensson, N. Core-Level Binding-Energy Shifts for the Metallic Elements. Phys. Rev. B 1980, 21, 4427-4457.

(29) Olovsson, W.; Marten, T.; Holmström, E.; Johansson, B.; Abrikosov, I. A. First Principle Calculations of Core-Level Binding Energy and Auger Kinetic Energy Shifts in Metallic Solids. J. Electron Spectros. Relat. Phenomena 2010, 178-179, 88-99.

(30) Cavallin, A.; Pozzo, M.; Africh, C.; Baraldi, A.; Vesselli, E.; Dri, C.; Comelli, G.; Larciprete, R.; Lacovig, P.; Lizzit, S.; et al., Local Electronic Structure and Density of Edge and Facet Atoms at Rh Nanoclusters Self-Assembled on a Graphene Template. ACS Nano 2012, 6, 3034-3043.

(31) Delesma, F.; den Bossche, M. V.; Grönbeck, H.; Calaminici, P.; Köster, A.; Pettersson, L. G. M. A Chemical View on X-Ray Photoelectron Spectroscopy: The ESCA Molecule and Surface-to-Bulk XPS Shifts. ChemPhysChem 2018, 19, 169.

(32) Baletto, F.; Ferrando, R. Structural Properties of Nanoclusters: Energetic, Thermodynamic, and Kinetic Effects. Rev. Mod. Phys. 2005, 77, 371-423.

(33) Posada-Borbón, A.; Heard, C. J.; Grönbeck, H. Cluster Size Effects in Ethylene Hydrogenation over Palladium. J. Phys. Chem. C 2017, 121, 10870-10875. 
(34) Andersen, J. N.; Hennig, D.; Lundgren, E.; Methfessel, M.; Nyholm, R.; Scheffler, M. Surface Core-Level Shifts of Some 4d-Metal Single-Crystal Surfaces: Experiments and Ab Initio Calculations. Phys. Rev. B 1994, 50, 17525.

(35) Brack, M. The Physics of Simple Metal Clusters: Self-Consistent Jellium Model and Semiclassical Approaches. Rev. Mod. Phys. 1993, 65, 677.

(36) de Heer, W. The Physics of Simple Metal Clusters: Experimental Aspects and Simple Models. Rev. Mod. Phys. 1993, 65, 611.

(37) Siegbahn, K. Electron Spectroscopy for Atoms, Molecules, and Condensed Matter. Rev. Mod. Phys. 1982, 54, 709.

(38) Gelius, U. Binding Energies and Chemical Shifts in ESCA. Phys. Scr. 1974, 9, 133.

(39) Lizzit, S.; Baraldi, A.; Groso, A.; Reuter, K.; Ganduglia-Pirovano, M. V.; Stampfl, . C.; Scheffler, M.; Stichler, M.; Keller, C.; Wurth, W.; Menzel, D. Surface Core-Level Shifts of Clean and Oxygen-Covered Ru(0001). Phys. Rev. B 2001, 63, 205319. 
Graphical TOC Entry

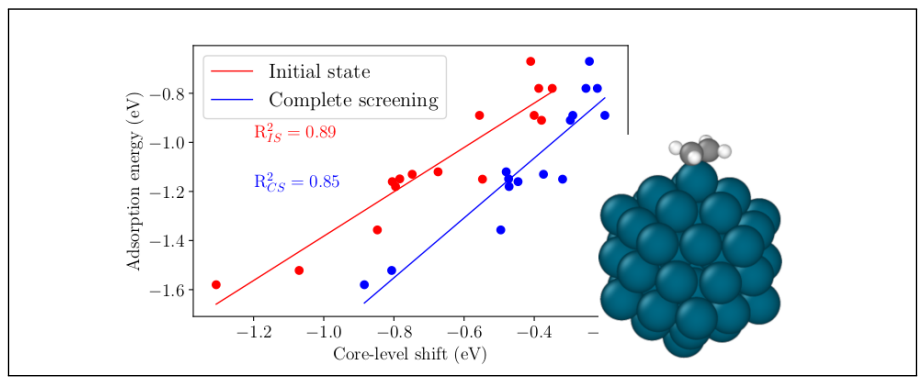

